\title{
Design of an Injectable in Situ Gelation Biomaterials for Vitreous Substitute
}

Annaka, Masahiko; Mortensen, Kell; Vigild, Martin E.; Matsuura, Toyoaki; Tsuji, Souichiro; Ueda, Tetsuo; Tsujinaka, Hiroki

\section{Published in:}

Biomacromolecules

Link to article, DOI:

$10.1021 / \mathrm{bm} 201012 \mathrm{f}$

Publication date:

2011

Document Version

Publisher's PDF, also known as Version of record

Link back to DTU Orbit

Citation (APA):

Annaka, M., Mortensen, K., Vigild, M. E., Matsuura, T., Tsuji, S., Ueda, T., \& Tsujinaka, H. (2011). Design of an Injectable in Situ Gelation Biomaterials for Vitreous Substitute. Biomacromolecules, 12(11), 4011-4021.

https://doi.org/10.1021/bm201012f

\section{General rights}

Copyright and moral rights for the publications made accessible in the public portal are retained by the authors and/or other copyright owners and it is a condition of accessing publications that users recognise and abide by the legal requirements associated with these rights.

- Users may download and print one copy of any publication from the public portal for the purpose of private study or research.

- You may not further distribute the material or use it for any profit-making activity or commercial gain

- You may freely distribute the URL identifying the publication in the public portal 


\title{
Design of an Injectable in Situ Gelation Biomaterials for Vitreous Substitute
}

\author{
Masahiko Annaka, ${ }^{*}{ }^{\dagger},+$ Kell Mortensen, ${ }^{\S}$ Martin E. Vigild, ${ }^{\|}$Toyoaki Matsuura, ${ }^{\perp}$ Souichiro Tsuji, ${ }^{\dagger}$ \\ Tetsuo Ueda, ${ }^{\perp}$ and Hiroki Tsujinaka ${ }^{\perp}$ \\ ${ }^{\dagger}$ Department of Chemistry, Kyushu University, Fukuoka 812-8581, Japan \\ ${ }^{\ddagger}$ International Research Center for Molecular Systems, Kyushu University, Fukuoka 819-0395, Japan \\ ${ }^{\S}$ Department of Natural Sciences, University of Copenhagen, Thorvaldsensvej 40, DK1871 Frederiksberg C, Denmark \\ ${ }^{\|}$Danish Polymer Center and Department of Chemical Engineering, Technical University of Denmark, DK-2800 Kgs. Lyngby, \\ Denmark \\ ${ }^{\perp}$ Department of Ophthalmology, Nara Medical University, Kashihara, Nara 634-8522, Japan
}

ABSTRACT: To adapt the physical properties of living materials to their biological function, nature developed various types of polymers with outstanding physical behavior. One example is the vitreous body, which is important intraocular elements not only because of its optical and mechanical performances, but also due to its important role in the pathogenesis and treatment of conditions affecting adjacent tissues and eventually the whole eye. Here, we report a novel biocompatible material for injectable vitreous substitute, composed of thermosensitive amphiphilic polymer, which is capable of forming a transparent gel in the vitreous cavity. It is nontoxic, provides adequate support for the retina, and allows light to reach the sensory elements at the back

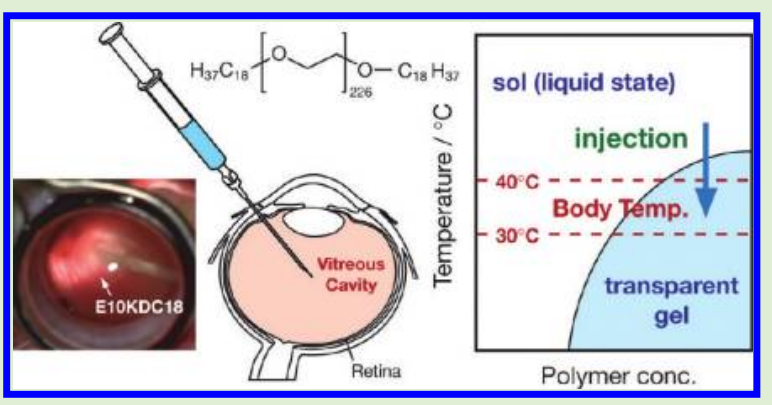
of the eye. The amphiphilic polymer exhibits mechanical stability by assembling to form highly interconnected hydrophobic domains, which leads to the constitution of a network structure.

\section{INTRODUCTION}

Very often nature combines different types of macromolecules to form gels with outstanding physical properties. One example is the vitreous body, which is a transparent jelly-like mass, composed of collagen and sodium hyaluronate, filling the posterior cavity of the eye. The vitreous body is important intraocular elements not only because of its optical and mechanical performances, but also due to its important role in the pathogenesis and treatment of conditions affecting adjacent tissues or eventually the whole eye. ${ }^{1,2}$ The vitreous body becomes dysfunctional either due to opacification or by its physical collapse and liquefaction. ${ }^{3}$ A collapsed or liquefied vitreous has a strong tendency to detach from the retina, and this change predisposes the retinal surfaces to damage, vitreous bleeding, or retinal detachment due to traction developing at any point of strong vitroretinal adhesion. All these conditions may result in extremely poor vision or even blindness. In principle, a total replacement of the afflicted vitreous body with biocompatible and transparent material would be desirable in such cases. It is the surgical treatment of various complicated retinal detachments that mostly requires the availability of a vitreous substitute, either temporary or permanent, ideally to be injectable into the cavity at the time of surgery.

New idea for the design of the vitreous substitute includes mimicking the natural vitreous by injection of elastic polymers into the vitreous cavity of the eye as a fluid. ${ }^{4,5}$ Body temperature transforms the polymer into a gel that completely fills the vitreous cavity. The gel does not leak, is entirely cohesive, and is expected to behave similar to the natural vitreous body.

Sodium hyaluronate and polysaccharides, such as dextran, alginic acid, chondroitin sulfate, guar gum, methylcellulose, carboxymethylcellulose, and hyroxypropylmethylcellulose, were studied as vitreous substitutes. ${ }^{6,7}$ Water-soluble high molecular weight polymers have rheological properties that permit the highly viscous solutions to be injected in the eye through small gauge needles. Nevertheless, the main defect of all these products is their short residence time in the vitreous cavity. In fact, they are reabsorbed in relatively short periods of time.

Silicone oil is the most commonly used temporary vitreous substitute. $^{8}$ However, the ability of the eye to tolerate this material has been repeatedly questioned. Different formulations, over the past decade, have been realized, but all of them can induce complications such as the disappearance of the outer plexiform layer and disorganization of the photoreceptor layer in eyes subjected to the treatment with silicone oils., 10 Some of the studies found that silicone oil may infiltrate various tissues of the eye. ${ }^{11,12}$ Since the 1980s, studies have reported

Received: July 21, 2011

Revised: September 20, 2011

Published: October 11, 2011 
the impregnation of the optic nerve with what was presumed to be silicone oil, both in front of and behind the lamia cribrosa. ${ }^{13,14}$ Furthermore, intraocular silicone oil, under special circumstance, may migrate into the brain through the subarachnoidal space. ${ }^{15}$ However, the most frequent event is the formation of an emulsification within the eye due to the infiltration of humor aqueous into the vitreous cavity containing the silicone oil. ${ }^{16}$

A hydrogel, therefore, should drastically decrease the risk of the emulsification once implanted into the vitreous cavity. Physical and chemical cross-linking techniques have been attempted to fill the vitreous cavity or to form gels in situ. Chilira et al. ${ }^{17-21}$ prepared a homopolymer of 1-vinyl-2pyrrolidone (VP) and its copolymer with 2-hydroxyethyl methacrylate (HEMA), both cross-linked with divinyl glycol, as possible substitutes for the vitreous body. The hydrogel behaved like viscoelastic gel, displaying excellent optical property. However, the material was found to be cytostatic. Half of the six rabbit eyes in which the gels were tested showed opacities that would inhabit vision. Other rabbits showed traction of the blood vessels with large white patches and cupping of the optic disk with haziness. The retina was intact, but choroidal veins and capillaries were dilated. Biodegradation of the polymer by phagocytosis occurred in vivo. Although selected cross-linked hydrogels showed mechanical properties close to that of the natural vitreous, the injection of the hydrogels resulted in physical fragmentation of the gel and severed entanglements, causing decreasing in the elasticity of the substitutes. While these substitutes appeared promising, they could not feasibly be injected through a small-gauge needle during surgery and retain mechanical properties.

Ravi et al. ${ }^{22}$ reported on an injectable hydrogel prepared by oxidation of an aqueous solution of acrylamide copolymers containing pendant thiol $(-\mathrm{SH})$ groups. Gelation was achieved through a thiol-disulfide exchange reaction by adding $3,3^{\prime}-$ dithiodipropionic acid. Although this reversible hydrogel system could circumvent monomer toxicity and heat of polymerization, it is still difficult to control cross-linking in the vitreous cavity.

Recently, Lamponi and co-workers ${ }^{23-25}$ prepared a poly(vinyl alcohol) (PVA) hydrogel using different amounts of trisodium trimetaphosphate (STMP) as cross-linking agent. They performed comprehensive characterization of the materials, including light transmittance, water content assessment, rheological measurements, and in vivo cytotoxicity assays. All these tests seemed to show that the hydrogel with a molar ratio of PVA/STMP of 8:1 fulfilled the requirements for a vitreous substitute. Due to their good optical properties and rheological features, PVA/STMP hydrogel is promising candidates for vitreous substitution, but further studies need to be performed to collect more data on their retention time in the eye, the long-term biocompatibility, mechanical properties, and ability on reattach the retina.

The triblock copolymer Pluronic F127 $\left(\mathrm{PEO}_{106}-\mathrm{PPO}_{70}-\right.$ $\left.\mathrm{PEO}_{106}\right)$ in aqueous solution is sol state at low temperatures, but forms a clear physical gel at physiological temperature. Its specific gravity is 1.032 and it has been used for slow drug delivery. Davidorf et al. ${ }^{26}$ evaluated Pluronic F127 as a vitreous substitute in nine rabbits. It was absorbed slowly from the rabbit vitreous in spite of its high molecular weight. It was injected into the vitreous of rabbits and the electroretinogram (ERG) amplitude decreased in the treated eyes within $24 \mathrm{~h}$ of surgery. There was an atrophy of photoreceptor outer segments with vacuolization of the retinal pigment epithelium after $24 \mathrm{~h}$. There was an obvious decrease in retinal function and toxicity of Pluronic F127. This would not be acceptable for clinical use. Rheologically, this material shows large shear thinning, with its viscosity significantly decreasing, not allowing injection into the eye. $^{17}$

Pritchard et al. ${ }^{27}$ tested the aqueous sols of poly(ethylene oxide) (PEO) as potential vitreous substitutes in a rabbit model. Sol of $5 \mathrm{wt} \%$ PEO with a molecular weight of $400000 \mathrm{~g}$ $\mathrm{mol}^{-1}$ in phosphate-buffered saline were shown to have mechanical and optical properties similar to the natural vitreous and were well tolerated by the retina, with minimal histological or electrophysiolosical changes, with the exception of glial fibrillary acidic protein (GFAP) up-regulation over a period of 41 days. However, the sols were not retained in the posterior body throughout the postoperative period. These results indicate that the use of a cross-linked PEO hydrogel as a potential artificial vitreous substitute.

For ophthalmic applications, the requirements for an in situ gel-forming system are stringent, including optically clear material very low toxicity, and long-term stability in a wet, oxygen-, and photon-rich environment. In this work, therefore, we focus on the development of a novel injectable vitreous substitute for intraocular applications, which is based on a thermosensitive hydrophobically modified poly(ethylene glycol) (HM-PEG). Among the various families of hydrophobically modified water-soluble polymers, telechelic derivatives bearing hydrophobic end groups have attracted attention over the decades, primarily because of their ability to form flowerlike micellar aggregates, which associate above a given polymer concentration, giving rise to highly viscoelastic fluids. The important class of polymeric additives known as hydrophobicethylene oxide-urethane (HEUR) copolymers are key components of numerous water-borne fluids, primarily because of their ability to induce viscoelastic properties in aqueous solutions. The characteristic feature of HEUR polymers in water is their ability to form flower micelles via attraction of the end groups. ${ }^{28,29}$ When the polymer concentration exceeds the overlap concentration, connectivity through bridging chains takes place, resulting in the formation of a network in which flower micelles act as junctions. This scenario described for the HEUR systems, turned out to be applicable also to the case of HM-PEG. Poly(ethylene glycol) end-capped with an octadecyl groups (E10KDC18) has the advantage to form gel that completely fills the vitreous cavity and provides the elastic modulus. The optical, toxicological, and histopathologic characteristics are also discussed.

\section{EXPERIMENTAL SECTION}

Materials. PEG $\left(M_{\mathrm{w}}=10000, M_{\mathrm{w}} / M_{\mathrm{n}}=1.15\right.$, Aldrich $)$ were purified by chromatography on activated alumina and lyophilized. Octadecyl bromide (Wako), sodium hydride (Wako), and 1,4-dioxane (Wako) were purified using standard methods.

Preparation of E10KDC18 Polymer. E10KDC18 were obtained via Williamson reaction of a octadecyl bromide on metalated PEG. PEG ( $5.0 \mathrm{~g} ; 0.50 \mathrm{mmol}$ ) was dissolved in $100 \mathrm{~mL}$ of 1,4-dioxane. Sodium hydride (purity $70 \%, 0.35 \mathrm{~g} ; 10.0 \mathrm{mmol}$ ) was added portionwise, and the mixture was stirred for $1 \mathrm{~h}$ under nitrogen. 1Bromooctadecane $(5.2 \mathrm{~mL} ; 15 \mathrm{mmol})$ was added dropwise. The solution was stirred for $24 \mathrm{~h}$ at room temperature under a nitrogen atomosphere. The crude product was precipitated by the dropwise addition to $2000 \mathrm{~mL}$ of diethylether. This precipitation process was repeated twice. The degree of substitution of the hydroxyl groups was determined by ${ }^{1} \mathrm{H}$ NMR and was found to be $100 \%$. 
Rheology. The viscoelastic properties were investigated by oscillatory measurements on the controlled Rheometrics RSA-II instrument, and samples $(0.2 \mathrm{~mm}$ in thickness) were mounted in a shear sandwich cell. A frequency sweep test was conducted with a shear amplitude $\gamma=1 \%$ under an oscillating frequency of $0.1-100 \mathrm{~s}^{-1}$.

Small-Angle X-ray Scattering. The small-angle X-ray scattering (SAXS) experiment was carried out with the BL-10 installed at Photon Factory (Tsukuba, Japan). An incident X-ray beam from the synchrotron orbital radiation was monochromatized to $1.49 \AA$. The scattered X-ray was detected with a one-dimensional position-sensitive proportional counter positioned at $1 \mathrm{~m}$ from the sample: the magnitude of the observed scattering vector ranged from 0.008 to $0.15 \AA^{-1}$. The sample was held in an aluminum holder sealed with two Kapton films, and the intensities were accumulated for $600 \mathrm{~s}$ to ensure sufficient statistical accuracy without degrading the gel samples by Xray irradiation.

Small-Angle Neutron Scattering. Small-angle neutron scattering experiments were performed at the SANS II facility installed at PSI, using $6 \AA$ neutrons with $9 \%$ wavelength resolution. The sample-todetector distance and the collimation length were both $6 \mathrm{~m}$. The samples were mounted in a shear sandwich cell in a Rheometrics RSAII instrument modified for the simultaneous measurements of both dynamic mechanical and structural properties and for studies of the structural response to large amplitude oscillatory shear. Large amplitude oscillatory shear used for the shear alignment studies were performed with oscillatory shear amplitude $g$ up to $160 \%$ at a frequency $\omega=1 \mathrm{~s}^{-1}$. The sample thickness was $0.2 \mathrm{~mm}$. The sandwich cell was mounted such that the shear velocity direction $(v)$ was vertical and the shear gradient $(\nabla)$ was horizontal and parallel to the neutron beam (Figure $8 \mathrm{a}$ ). When viewing a two-dimentional diffraction image obtained in this conformation, the shear velocity is in the vertical direction, and the horizontal direction is parallel to the velocity vector (e).

Transmission. Transmission of light was measured using a JASCO V-660 spectrophotometer within the range of $200-800 \mathrm{~nm}$ at $37{ }^{\circ} \mathrm{C}$ with an accuracy of $\pm 0.1{ }^{\circ} \mathrm{C}$.

Refractive Index. The refractive index was measured with an ATAGO DR-M2/1550 refractometer. For all measurements, the temperature was kept at $37{ }^{\circ} \mathrm{C}$ with an accuracy of $\pm 0.1{ }^{\circ} \mathrm{C}$.

Transport Property. The anterior segment (cornea, iris, and rens) of a pig eye was removed. A retinal detachment with a hole was created in the pig eyecup. One of the eyecups was filled with 25 wt \% E10KDC18 gel, and the other was used as a control by filling with saline. Applying $0.3 \mathrm{~mL}$ of dilute aqueous solution of methylene blue to eyecups allowed us to observe the transport property of the E10KDC18 gel network.

Cytotoxicity. A murine catecholaminergic cell line derived from the B6/D2 F1 mouse, CATH.a, were seeded in a six-well culture dishes at the density of $1 \times 10^{4}$ cells per well in Dulbecco's modified Eagle's medium (DMEM, SIGMA) supplemented with $10 \%$ fetal bovine serum (FBS, GIBCO/BRL), $50 \mathrm{U} / \mathrm{mL}$ penicillin, and $50 \mathrm{mg} /$ $\mathrm{mL}$ streptomycin (GIBCO). The cells were incubated at $37{ }^{\circ} \mathrm{C}$ in a humidified $5 \% \mathrm{CO}_{2}$ atmosphere. After 7 days of culture, the medium in the wells was replaced with the fresh medium containing of varying concentration of E10KDC18 polymer (2.0, 5.0, 7.5, and 10 wt \%). After 2 days, cell viability was assessed by trypan blue exclusion.

Surgical Procedures. The surgeries were performed under general anesthesia with intramuscle injection of pentobarbital sodium $(20 \mathrm{mg} / \mathrm{kg})$. All studies were conducted in accordance with the Association for Research in Vision and Ophthalmology (ARVO) Resolution on the Use of Animals in Vision and Ophthalmic Research. Under an operating microscope, each animal underwent pars plana vitrectomy with two port systems, port size 21 gauge, at $2.5 \mathrm{~mm}$ from the limbus. The vitreous body was removed as much as possible and replaced with air. Then, each sample was injected into the vitreous cavity. Two port wounds were closed with 8-0 polypropylene suture and treated with cryopexy $\left(\mathrm{NO}_{2}\right)$ for $7 \mathrm{~s}$. After surgery, the eyes were treated with Levofloxacin as antibiotic eyedrops and betamethasone sodium phosphate as steroid eyedrops three times a day for 1 week.

\section{RESULTS AND DISCUSSION}

Hydrophobically end-capped poly(ethylene glycol) with two aliphatic end groups $(\alpha, \omega$-PEG) belongs to the family of the associative polymers ${ }^{30-32}$ and was selected as the main element for the vitreous body substitute: when dissolved in water, hydrophobic ends associate into microdomains to form spherical micelles, which are expected to form a gel for concentrated solution. $\alpha, \omega$-PEG with molar mass $M_{\mathrm{w}}=10 \mathrm{kD}$ (E10K) were prepared by functionalization of the terminal hydroxyl groups on both ends of PEG with octadecyl groups, C18 (DC18), giving the polymer abbreviated E10KDC18.

As shown in Figure 1a, we distinguished macroscopically, with changes in the temperature or concentration, three regions

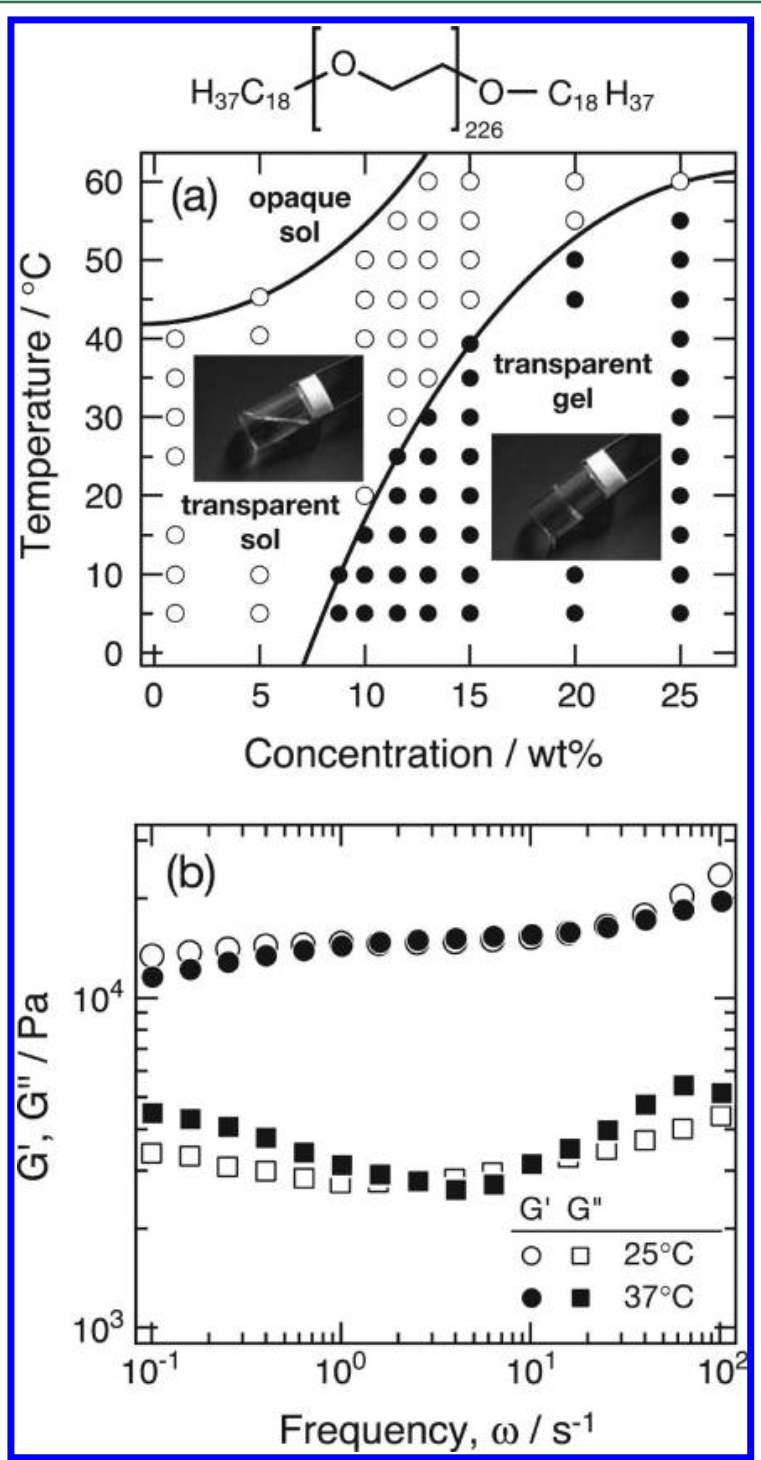

Figure 1. (a) Chemical structure and phase diagram for E10KDC18 polymer in water. Phase boundary is determined by visual observation. (b) Dynamic elastic moduli for the $25 \mathrm{wt} \%$ gel sample as a function of frequency (measured with a cone and plate geometry) at 25 and 37 ${ }^{\circ} \mathrm{C}$. These data is typical of the gel samples in that elastic storage modulus $G^{\prime}$ is substantially greater than the viscous loss modulus $G^{\prime \prime}$ over three decades of frequency measured.

in the phase diagram for aqueous solution of E10KDC18 in weight: transparent sol, opaque sol, and transparent gel. These changes occurred reversibly, without hysteresis, when the 
temperature is decreased. The storage modulus $G^{\prime}$ and the loss modulus $G^{\prime \prime}$ for 25 wt \% E10KDC18 polymer in water at 25 and $37{ }^{\circ} \mathrm{C}$ showed behavior typical of the gel samples in that the elastic storage modulus $G^{\prime}$ was substantially greater than the viscous loss modulus $G^{\prime \prime}$ over the full range of frequency measured (Figure 1b). As a function of the E10KDC18 polymer concentration, the $G^{\prime} / G^{\prime \prime}$ ratio varied between 3 and 5 in the gel phase. Gels resist shear, yielding a reproducible response; frequency data for a gel sample before and after a dynamic stress sweep test are essentially unchanged.

The self-assembly of E10KDC18 polymers in heavy water was examined by small-angle neutron scattering (SANS) and by small-angle X-ray scattering (SAXS). We have made studies along lines of increasing E10KDC18 polymers concentration, as obtained at fixed temperature, and versus temperature for fixed polymer concentration. Figure 2 shows experimental SANS

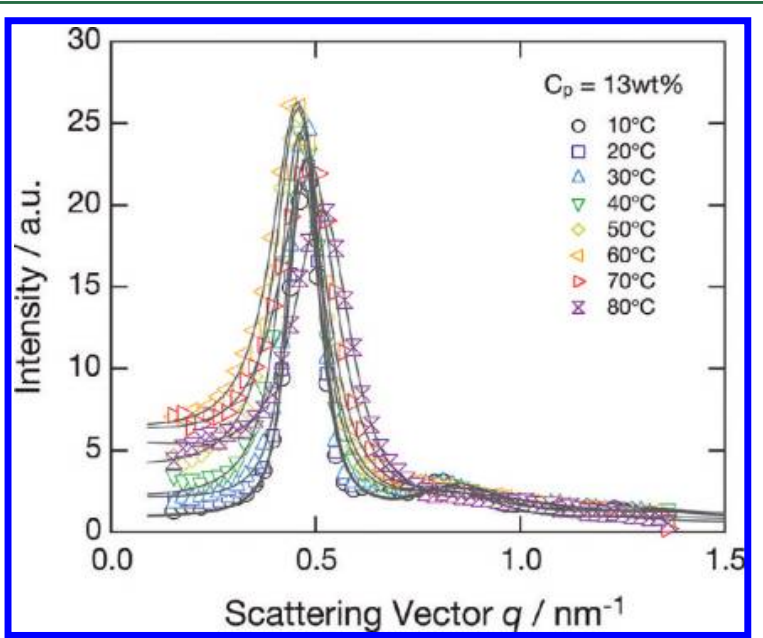

Figure 2. SANS scattering data of 13 wt \% E10KDC18 polymers in heavy water suspensions, as measured at temperatures within the range from 10 to $80{ }^{\circ} \mathrm{C}$. Solid lines are the best fit to the hard-sphere interacting micellar model described in the text.

data of 13 wt \% E10KDC18 polymers in heavy water suspensions, as measured within the temperature range from 10 to $80{ }^{\circ} \mathrm{C}$. The data are all characterized by a pronounced correlation peak close to $q=0.5 \mathrm{~nm}^{-1}$. The SANS structural data are fairly well fitted to the model of hard-sphere interacting polymeric micelles,

$$
I(q)=K P_{\mathrm{mic}}(q) S_{\mathrm{hs}}(q)
$$

where $q$ is the length of scattering vector, and the micellar form factor, $P_{\text {mid }}$ is given by ${ }^{33}$

$$
\begin{aligned}
& P_{\text {mic }}(q)=N_{\text {agg }}^{2} \beta_{\mathrm{s}}^{2} P_{\mathrm{s}}(q, R) \\
& \quad+N_{\mathrm{agg}} \beta_{\mathrm{c}}^{2} P_{\mathrm{c}}\left(q, R_{\mathrm{g}}\right)+N_{\mathrm{agg}}\left(N_{\mathrm{agg}}-1\right) \beta_{\mathrm{c}}^{2} S_{\mathrm{cc}}(q) \\
& \quad+2 N_{\mathrm{agg}}^{2} \beta_{\mathrm{s}} \beta_{c} S_{\mathrm{sc}}(q)
\end{aligned}
$$

where $N_{\text {agg }}$ is the micellar aggregation number, $\beta_{\mathrm{s}}$ and $\beta_{\mathcal{o}}$ respectively, are the total excess scattering length of blocks in the core and in the chain, and $R_{c}$ the radius of the micellar core, which is described by the spherical form factor

$$
\begin{aligned}
P_{\mathrm{s}}\left(q, R_{\mathrm{c}}\right) & =\Phi\left(q, R_{\mathrm{c}}\right)^{2} \\
& =\left[\frac{3\left[\sin \left(q R_{\mathrm{c}}\right)-q R_{\mathrm{c}} \cos \left(q R_{\mathrm{c}}\right)\right]}{\left(q R_{\mathrm{c}}\right)^{3}}\right]^{2}
\end{aligned}
$$

The corona polymer chains are characterizes by the radius of gyration, $R_{\mathrm{g}}$ and the form factor

$$
P_{\mathrm{c}}\left(q, R_{\mathrm{g}}\right)=\frac{2\left[\exp \left[\left(q R_{\mathrm{g}}\right)^{2}\right]-1+\left(q R_{\mathrm{g}}\right)^{2}\right]}{\left(q R_{\mathrm{g}}\right)^{4}}
$$

The cross-correlation term between the micellar core and the corona chains attached to the surface of the core, $S_{\mathrm{sc}}(q)$ is given by

$$
S_{\mathrm{sc}}(q)=\Phi(q, R) \psi\left(q, R_{\mathrm{g}}\right) \frac{\sin \left(q R_{\mathrm{c}}\right)}{q R_{\mathrm{c}}}
$$

where $\Phi(q, R)$ is the spherical form factor amplitude given in eq 3 , while $\psi\left(q, R_{\mathrm{g}}\right)$

$$
\psi\left(q, R_{\mathrm{g}}\right)=\frac{1-\exp \left[-\left(q R_{\mathrm{g}}\right)^{2}\right]}{\left(q R_{\mathrm{g}}\right)^{2}}
$$

is the chain form factor amplitude. The cross-correlation term between the two different chains in the corona, $S_{c c}(q)$, is correspondingly given by

$$
S_{\mathrm{cc}}(q)=\psi\left(q, R_{\mathrm{g}}\right)^{2}\left[\frac{\sin \left(q R_{\mathrm{c}}\right)}{\left(q R_{\mathrm{c}}\right)}\right]
$$

The structure factor describing intermicellar correlations is calculated using the Percus-Yevick approximation with hardsphere interaction potential: ${ }^{34}$

$$
S_{\mathrm{hs}}\left(q, R_{\mathrm{hs}}\right)=\frac{1}{1+24 \phi G\left(2 q R_{\mathrm{hs}}, \phi\right) /\left(2 q R_{\mathrm{hs}}\right)}
$$

$G\left(2 q R_{\mathrm{hs}}, \phi\right)$ is a trigonometric function of volume fraction, $\phi$, and $R_{\mathrm{hs}}$ being the hard-sphere interaction radius.

Figure 2 shows experimental data and best fits to the model, using standard least-squares fit method. The resulting parameters are the micellar core radius, $R_{\mathcal{c}}$ the corona polymer size, $R_{\mathrm{g}}$, the interaction radius, $R_{\mathrm{hs}}$, and the micellar volume fraction, $\phi$. The corona size, expressed by the polymer radius of gyration, $R_{\mathrm{g}}$, is of the order of $3 \mathrm{~nm}$, independent of temperature. The interaction radius, $R_{\mathrm{hs}}$, changes gradually from $7.5 \mathrm{~nm}$ at $10{ }^{\circ} \mathrm{C}$ to slightly more than $6 \mathrm{~nm}$ at $80{ }^{\circ} \mathrm{C}$.

Both the micellar core size and the volume fraction, shown in respectively Figure $3 a$ and $b$, shows distinct temperature dependence, in particular within the $25-35^{\circ} \mathrm{C}$ range, likely as result of a sol-gel transition induced by a hard-sphere crystallization. At temperatures below approximately $30{ }^{\circ} \mathrm{C}$ the volume fraction is relative constant of the order of 0.54 , that is, larger than the critical value (0.48) for hard-sphere crystallization, in agreement with our findings of body-centered cubic (bcc)-ordered structure, as discussed below. Close to $T \approx$ $30{ }^{\circ} \mathrm{C}$, a marked change in apparent volume fraction appears, signifying the transformation from the low-temperature micellar ordered state to high-temperature micellar liquid.

Figure 4 shows experimental SANS data of E10KDC18 polymers in heavy water suspensions within the concentration range $1-25 \mathrm{wt} \%$, as measured at $25{ }^{\circ} \mathrm{C}$. Except for the $1 \%$ 


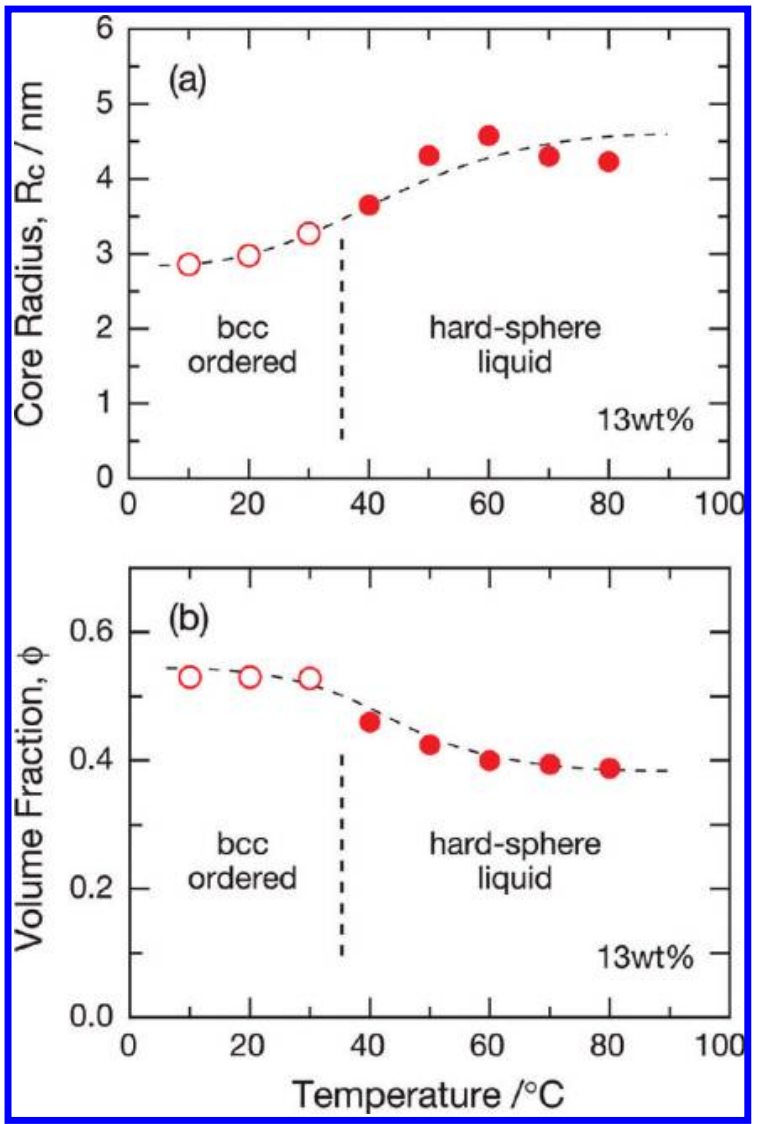

Figure 3. (a) Temperature-dependence of micellar core size of $13 \mathrm{wt}$ $\% \mathrm{E} 10 \mathrm{KDC} 18$ polymers in water suspensions, as resulting from fits to the SANS data displayed in Figure 2. Dashed line is a guide to the eye. (b) Temperature-dependence of micellar volume fraction of $13 \mathrm{wt} \%$ E10KDC18 polymers in water suspensions, as resulting from fits to the SANS data displayed in Figure 2. Dashed line is a guide to the eye.

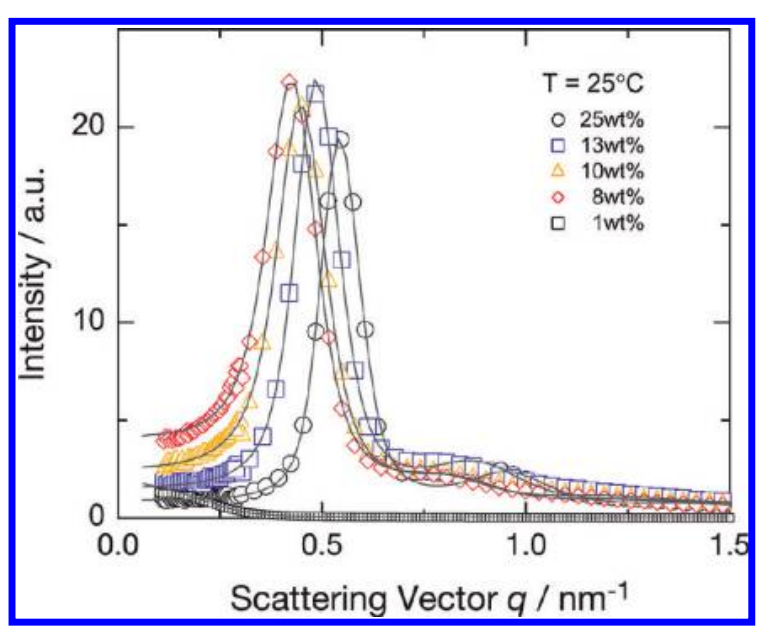

Figure 4. SANS scattering data of E10KDC18 polymers in heavy water suspensions, within the concentration range from 1 to $25 \%$. Solid lines are fits to hard-sphere interacting micelles (see text).

sample, the data are all characterized by a pronounced correlation peak within the $q$ range $0.4-0.6 \mathrm{~nm}^{-1}$. The $1 \%$ sample shows the characteristics of noninteracting micelles, with size of the order of $8 \mathrm{~nm}$. The SANS structural data are fairly well fitted to the model of hard-sphere interacting polymeric micelles, eq 1 . The fits are very good for concentrations below approximately $13 \%$, while the 13 and
$25 \%$ samples show some systematic deviation between data and fit at intermediate $q$-values (see also Figure 6). The corona size, $R_{\mathrm{g}}$, and the interaction radius, $R_{\mathrm{hs}}$, are both roughly independent of polymer concentration, being of the order of, respectively, 3 and $7 \mathrm{~nm}$. The core size shows distinct concentration dependence, as seen in Figure 5a. The volume

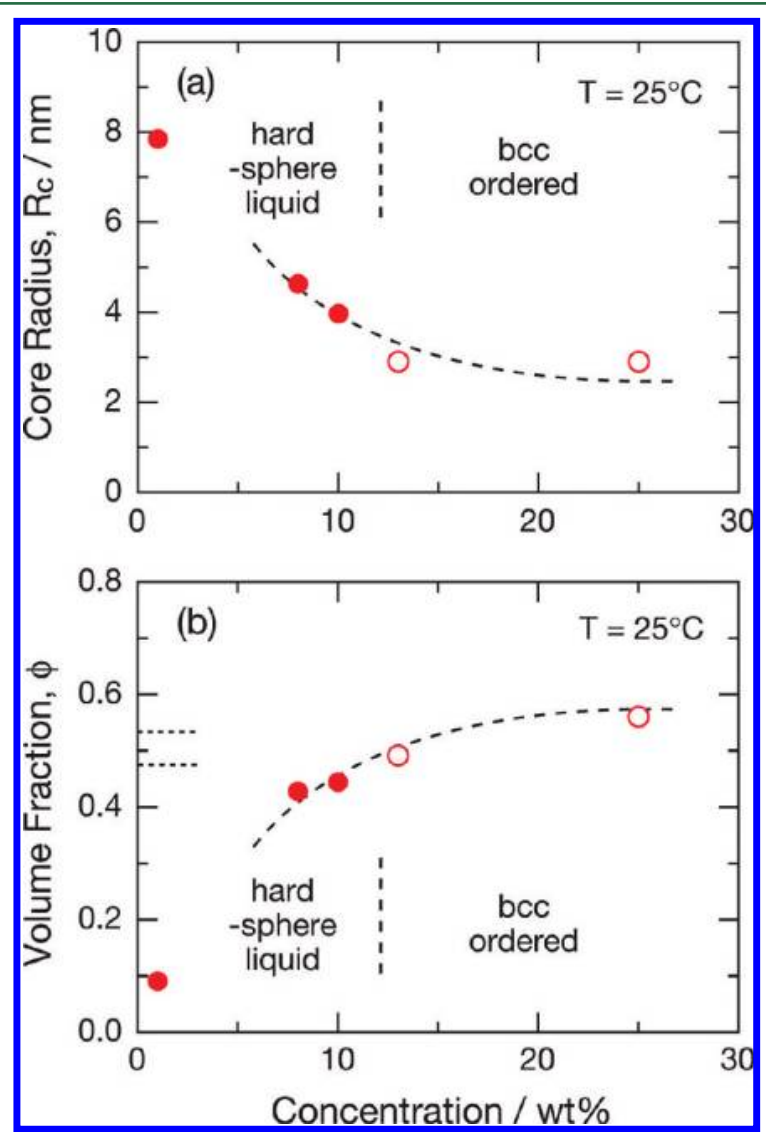

Figure 5. (a) Concentration dependence of micellar core size of E10KDC18 polymers in water suspensions, as resulting from fits to the SANS data displayed in Figure 2. Dashed line is a guide to the eye. (b) Concentration dependence of micellar volume fraction of E10KDC18 polymers in water suspensions, as resulting from fits to the SANS data displayed in Figure 2. Dashed line is a guide to the eye. For concentrations of $13 \%$ and above, the volume fraction is larger than the critical value for hard-sphere crystallization. The scattering data show at these concentrations evidence of bcc-ordered micelles.

fraction, shown in Figure 5b, also displays significant dependence on concentration. For concentrations beyond approximately $13 \%$, the volume fraction is larger than the critical value for hard-sphere crystallization. The scattering function is, in agreement with this, not really well described by the structure factor of hard-sphere interacting micelles.

In Figure 6 is shown the $25 \%$ data, and best fits to the hardsphere model and best fits to similar micellar system, but ordered on a bcc lattice, that is, the form factor is described by eq 2, while the structure factor is in accordance with a bcc lattice with a correlation length that is one of the fitting parameters. The experimental data are not in full agreement with either of the two models. The hard-sphere liquid cannot account for the higher scattering at $q \approx 0.7 \mathrm{~nm}^{-1}$, which on the other hand is well described by the (111)-Brag peak of the bccordered system. Neither the micellar form factor nor polydispersity can, however, account for the detailed form 


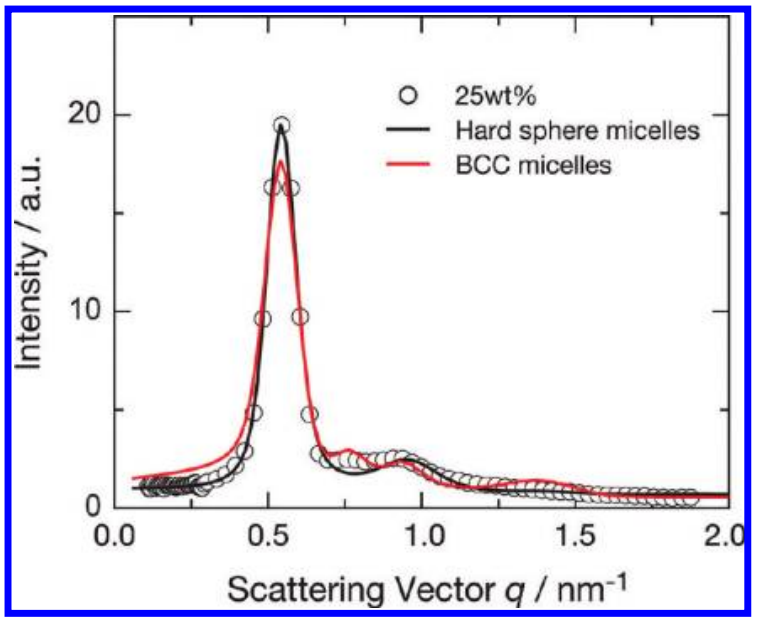

Figure 6. SANS scattering data of $25 \%$ E10KDC18 polymers in heavy water suspensions. Dotted line is the best fit to the hard-sphere interacting micellar model described in the text, while the solid line represent polymer micelles positioned at a bcc lattice.

and intensity of the experimentally observed higher-order Bragg peak. We therefore conclude that the $25 \%$ systems constitute a mixture of bcc-ordered and hard-sphere liquid of polymer micelles. The volume fraction resulting from fits to the experimental data ( 0.49 for $13 \%$ and 0.55 for $25 \%$ ) are in accordance with this conclusion: According to prior observations in micellar suspensions ${ }^{35}$ and colloids, ${ }^{36}$ the phase is characterized as coexisting regime for volume fractions within the $0.48-0.54$ regime.

Figure 7 shows experimental SAXS data obtained for E10KDC18 polymers within the $10-25$ wt \% concentration

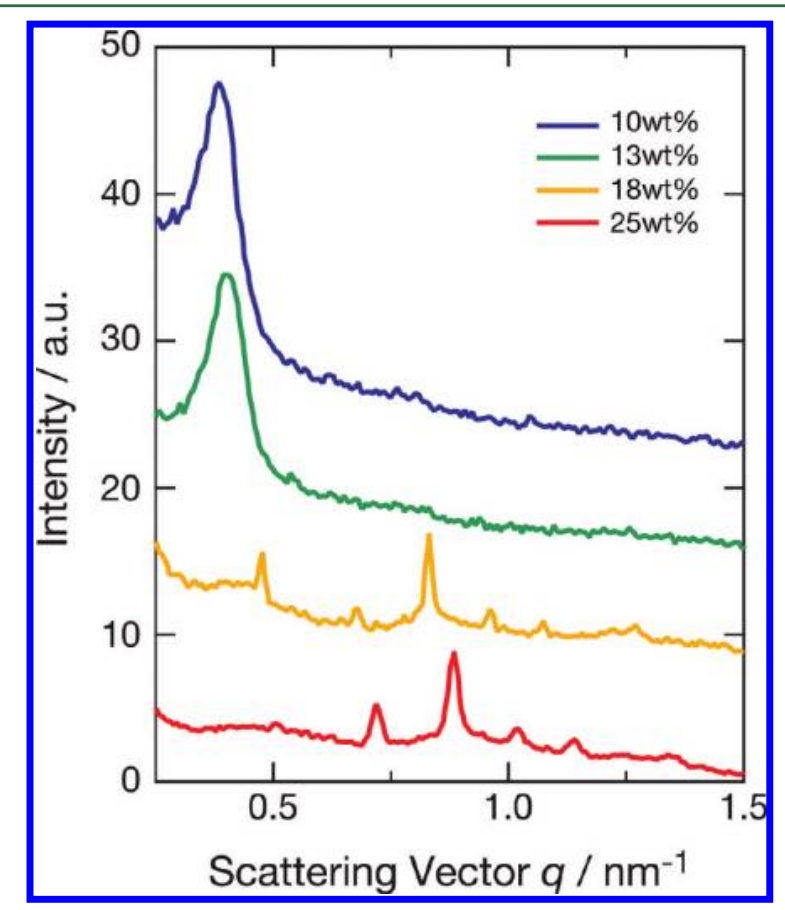

Figure 7. SAXS data on four samples along a line of increasing E10KDC18 polymer concentration in water: $C_{\mathrm{p}}=10,15,20$, and 25 wt \%. First two samples are in sol regime, and the last two samples are in gel regime. The peaks for $20 \mathrm{wt} \%$ sample indicate planes corresponding to bcc cubic lattice. range, measured at $T=25{ }^{\circ} \mathrm{C}$. The SAXS data confirm high concentration ordered structure, showing a number of wellresolved Bragg peaks for concentrations beyond $15 \%$. The SAXS patterns are consistent with micelles packed in a bcc lattice with lattice constants of $185 \AA$ for the $20 \mathrm{wt} \%$ gel and $175 \AA$ for the $25 \mathrm{wt} \%$ gel. The self-association of octadecyl groups not only promotes the formation of highly interconnected end-group domains but also constitutes a network with bcc microstructure (Figure $5 b$ ). The marked changes in relative intensity of the different Bragg peaks confirm the changes in micellar sizes, as concluded from the SANS analysis.

From the structural viewpoint, the vitreous is a fascinating result of natural evolution as the heterotypic collagen fibers forms contiguous networks through indirect interactions and provide a rigid scaffold, while the hyaluronan macromolecules impart shock-absorbing properties to the whole system. The concentration of hyaluronan increases from the anterior portion near the lens to posterior vitreous by the retina, which is different from the structure E10KDC18 gel revealed by SANS and SAXS experiments in this study. ${ }^{37-41}$ However, the detailed structure of the natural vitreous and the reason for the heterogeneity of the natural vitreous are still unknown. The goal of this study, therefore, is to improve on current vitreous substitutes by mimicking the physicochemical and optical properties of natural vitreous. It is not necessary to match the structure, composition and physiology of the natural vitreous as well. Therefore, homogeneous synthetic hydrogels are being evaluated as vitreous substitutes because they are capable of matching the physicochemical and optical properties of natural vitreous humor.

The mechanical energy generated at high frequencies by external forces acting on the vitreous body, such as rubbing and hitting, and by heartbeats and eye movements, needs to be stored first and then dissipated slowly. As has already been shown in Figure 1b, E10KDC18 gel exhibits a $G^{\prime}$ greater than $G^{\prime \prime}$ and their values are relatively frequency insensitive. We also investigated structural response of the gel to large-amplitude oscillatory shear by simultaneous measurements of small-angle neutron scattering and rheology for $25 \mathrm{wt} \%$ gel for the shear frequency $\omega=1 \mathrm{~s}^{-1}$ at $25^{\circ} \mathrm{C}$. $^{42-48}$ The shear amplitude $\gamma$ was increased stepwise from $1 \%$ up to $100 \%$ and then the sample was allowed to relax back to a quiescent state (with measurements of the dynamic moduli at $\gamma=1 \%$ ). The results are plotted as the dynamic shear moduli $\left(G^{\prime}\right.$ and $\left.G^{\prime \prime}\right)$ as a function of time with scattering patterns corresponding to particular regimes: $\gamma=1,10,50,80,100 \%$ and the relaxed state in Figure $8 \mathrm{~b}$. The bcc microstructure aligns into different textures on a lattice. The scattering pattern obtained with low strain amplitude exhibits the liquid-like profile, namely, azimuthally isotropic and correlation peak centered at $q^{*}=$ $0.42 \mathrm{~nm}^{-1}$, and the hint of the second-order reflection at $2^{1 / 2} q^{*}$ from the disordered cubic structure. The symmetry of the SANS patterns, however, was unaltered up to $\gamma=10 \%$ (Figure $8 \mathrm{c}-\mathrm{i})$, over which range a transition to a highly ordered textures was observed. The onset of nonlinear response is apparent above $50 \%$ strain. Simultaneously with the start of the decrease in elastic moduli, texture starts to appear in the scattering patterns, suggesting stress relaxation due to the slip along given crystallographic orientations. At $\gamma=50 \%$, the loss modulus $G^{\prime \prime}$ was larger than the storage modulus $G^{\prime}$, and both $G^{\prime}$ and $G^{\prime \prime}$ decreased as the strain was increased, showing that the gel was shear thinning, and a 6-fold symmetric pattern emerges (Figure $8 \mathrm{c}$-ii). This scattering pattern can be associated with a bcc 


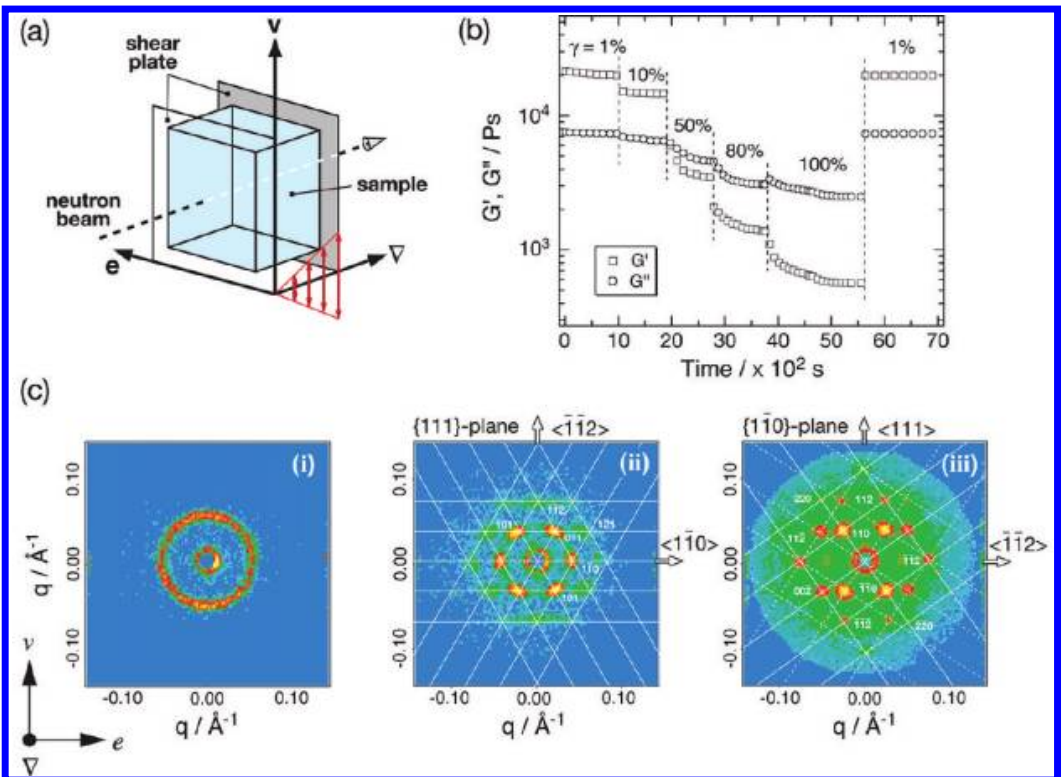

Figure 8. (a) Schematic diagram of the flow geometry and the scattering setup used for in situ rheology-SANS measurements; (b) shear moduli ( $G^{\prime}$ and $\left.G^{\prime \prime}\right)$; and (c) scattering pattern, as obtained simultaneously during large-amplitude oscillatory shear and after relaxation for shear amplitude in the range $\gamma=1-100 \%$, measured with fixed shear frequency $\omega=1 \mathrm{~s}^{-1}$ and temperature $T=25^{\circ} \mathrm{C}$.

structure with $\{111\}$ sliding planes and shear velocity $(\bar{\nu})$ coinciding with the $\langle\overline{11} 2\rangle$ direction. Here $\{h k l\}$ and $\langle h k l\rangle$ represent the crystal plane and crystal axis parallel to the $(\bar{\nu} \bar{\nabla})$ shear plane and $\bar{\nu}$ shear direction, respectively. This gives the vorticity vector $(\bar{e})$ parallel to the $\langle 1 \overline{1} 0\rangle$ direction of the bcc domain and the shear gradient $(\bar{\nabla})$ parallel to $\langle 111\rangle$ direction. It is not possible, however, from this characteristic 6-fold symmetric scattering pattern to judge whether the sample is a true single-domain oriented $\{111\} /\langle\overline{11} 2\rangle$ crystal or possibly a twin structure. According to the recent detailed crystallographic studies on the bcc morphologies of shear-aligned block copolymer systems, ${ }^{44-48}$ the observed 6-fold scattering pattern emerged by shear is interpreted to be a simple $90^{\circ}$ rotation of the domain structure observed at a higher shear mentioned below, that is, the $\{1 \overline{1} 0\} /\langle 111\rangle+\{\overline{1} 10\} /\langle 111\rangle$ twin rotated $90^{\circ}$ around the common $\langle 111\rangle$ flow axis, resulting in the $\{11 \overline{2}\} /\langle 111\rangle+\{\overline{11} 2\} /\langle 111\rangle$ orientation, for which sliding occurs within the twin boundaries. The scattering patterns obtained at high strain amplitude $\gamma>80 \%$ and relaxation after cessation of shear show well-resolved twinned bcc morphology (Figure $8 \mathrm{c}$-iii). In the twin structure, the crystalline slip system is $\{1 \overline{1} 0\} /\langle 111\rangle+\{\overline{1} 10\} /\langle 111\rangle$. The $\{110\}$ planes are forced to stack normal to the shear gradient and slide relative to each other with a common $\langle 111\rangle$ direction parallel to the shear flow $\bar{\nu}$. The boundary between the twins is a common $\{\overline{11} 2\}$ plane normal to the neutral direction of shear, $\bar{e}$. The shear-induced alignment of the random-domain structure, therefore, provides sufficient mechanical stability. A remarkable finding is that the cessation of shear led to the rapid recovery of the original values of $G^{\prime}$ and $G^{\prime \prime}$ and the twin bcc scattering pattern continues to exist in the relaxed state. This indicates that the large oscillatory shear induced a change in alignment of the cubic structure that was retained on cessation of shear, although the rheological properties in a quiescent state were not affected by this symmetry change.

Temperature sweep measurement of $G^{\prime}$ and $G^{\prime \prime}$ for the E10KDC18 was performed at the heating and cooling rate of 1 ${ }^{\circ} \mathrm{C} \mathrm{min}^{-1}$ and a constant frequency of $1 \mathrm{~s}^{-1}$. The sample was heated from 20 to $80^{\circ} \mathrm{C}$. The temperature sweep of $G^{\prime}$ and $G^{\prime \prime}$ for the E10KDC18 gel is presented in Figure 9. With raising

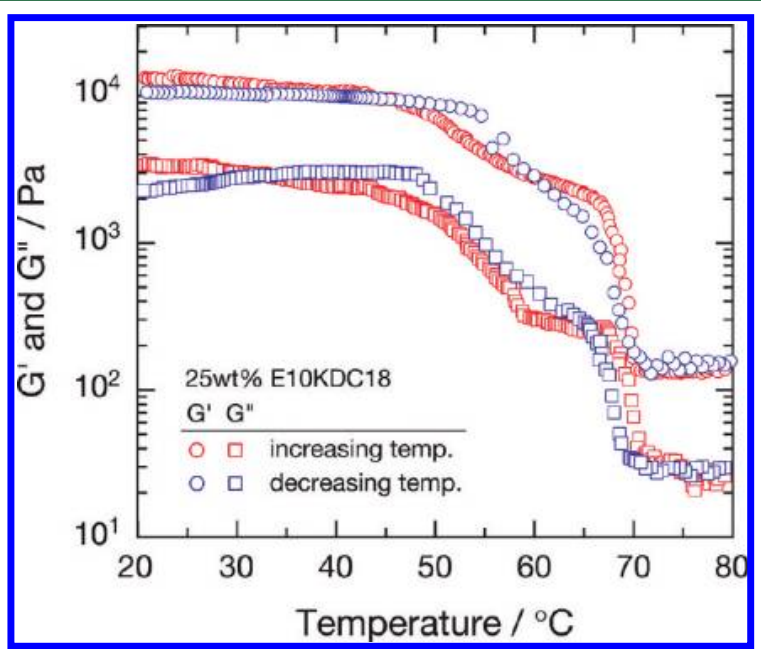

Figure 9. Temperature dependence of dynamic moduli $\left(G^{\prime}\right.$ and $\left.G^{\prime \prime}\right)$ of 25 wt \% E10KDC18 gel during isochronal dynamic temperature sweep experiments in heating and cooling process with $1{ }^{\circ} \mathrm{C} \mathrm{min}^{-1}$ for temperature ranging from 20 to $80{ }^{\circ} \mathrm{C}$ measured with fixed shear frequency $\omega=1 \mathrm{~s}^{-1}$ and shear amplitude $\gamma=1 \%$.

temperature, the moduli start to decrease at $45{ }^{\circ} \mathrm{C}$. On a further increase in temperature, the moduli decrease sharply at approximately $65{ }^{\circ} \mathrm{C}$, and $G^{\prime}$ and $G^{\prime \prime}$ values at $70{ }^{\circ} \mathrm{C}$ are 2 orders of magnitude smaller than those for ambient temperature. Decreasing the temperature after an annealing period of 5 min at $80^{\circ} \mathrm{C}$, despite small hysteresis, the rheological behavior of the E10KDC18 gel is essentially thermoreversible. The moduli at $20{ }^{\circ} \mathrm{C}$ are of the same magnitude before and after heating the sample to $80^{\circ} \mathrm{C}$.

It is preferred that the mechanical properties of the gel remain unchanged in the vitreous cavity after injection. In Figure 10, the storage modulus $G^{\prime}$ and the loss modulus $G^{\prime \prime}$ of 


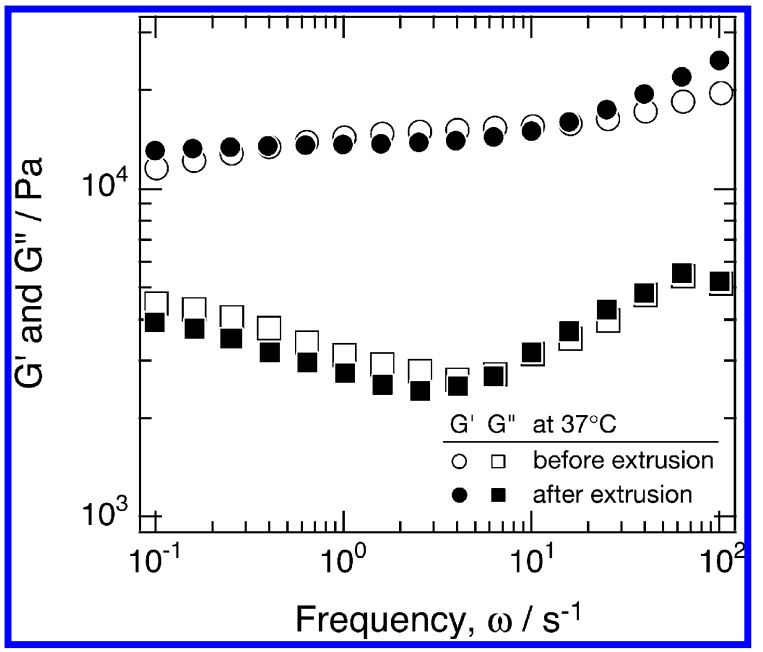

Figure 10. Comparison of the dynamic elastic moduli for the $25 \mathrm{wt} \%$ E10KDC18 gel before and after extrusion through 21-gauge cannula as a function of frequency (measured with a cone and plate geometry) at $\gamma=1 \%$ and $37{ }^{\circ} \mathrm{C}$.

the 25 wt \% E10KDC18 gel before and after extruding through a 21-gauge cannula at $37^{\circ} \mathrm{C}$ are compared as a function of the applied frequency. The gel shows same rheological behavior before and after extrusion, indicating the injection does not affect the network structure of the E10KDC18 hydrogel. These features of the polymer indicate that E10KDC18 gel demonstrates the feasibility of the development of an injectable in situ gelation biomaterial.

The refractive index of the 25 wt \% E10KDC18 gel in water at $37^{\circ} \mathrm{C}$ was 1.353 , which is not greatly different from the value of the natural vitreous body (1.336). ${ }^{49}$ As shown in Figure 11,

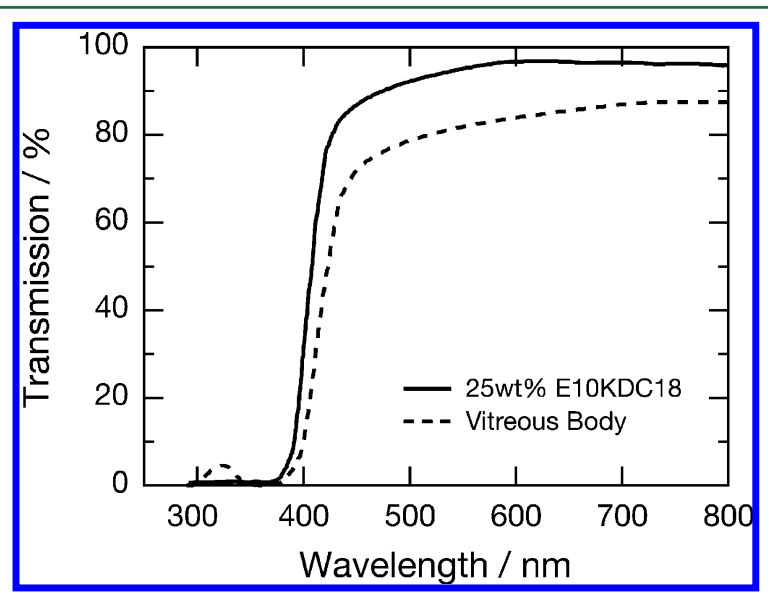

Figure 11. Transmission of light of the human vitreous body and 25 wt \% E10KDC18 gel as a function of wavelength ranging from 250 to $800 \mathrm{~nm}$ at $37^{\circ} \mathrm{C}$.

the 25 wt \% E10KDC18 gel has a reasonable transmission ( $>90 \%$ ranging from 400 to $800 \mathrm{~nm}$ ), which is comparable to the human vitreous body and filters out most of the UV light. The optical clarity has been maintained in vitro. It is worthy to mention that the transmission did not change even after largeamplitude oscillatory shear was applied to the gel, because the characteristic scale of the ordered structure is smaller than the wavelength of visible light.

Quantitative concentration-toxicity relationships were determined for murine catecholaminergic cells with several concentrations of E10KDC18 polymer. $^{50}$ The general morphology of the cells incubated with E10KDC18 polymer after staining with trypan blue is shown in Figure 12a. ${ }^{51}$ The

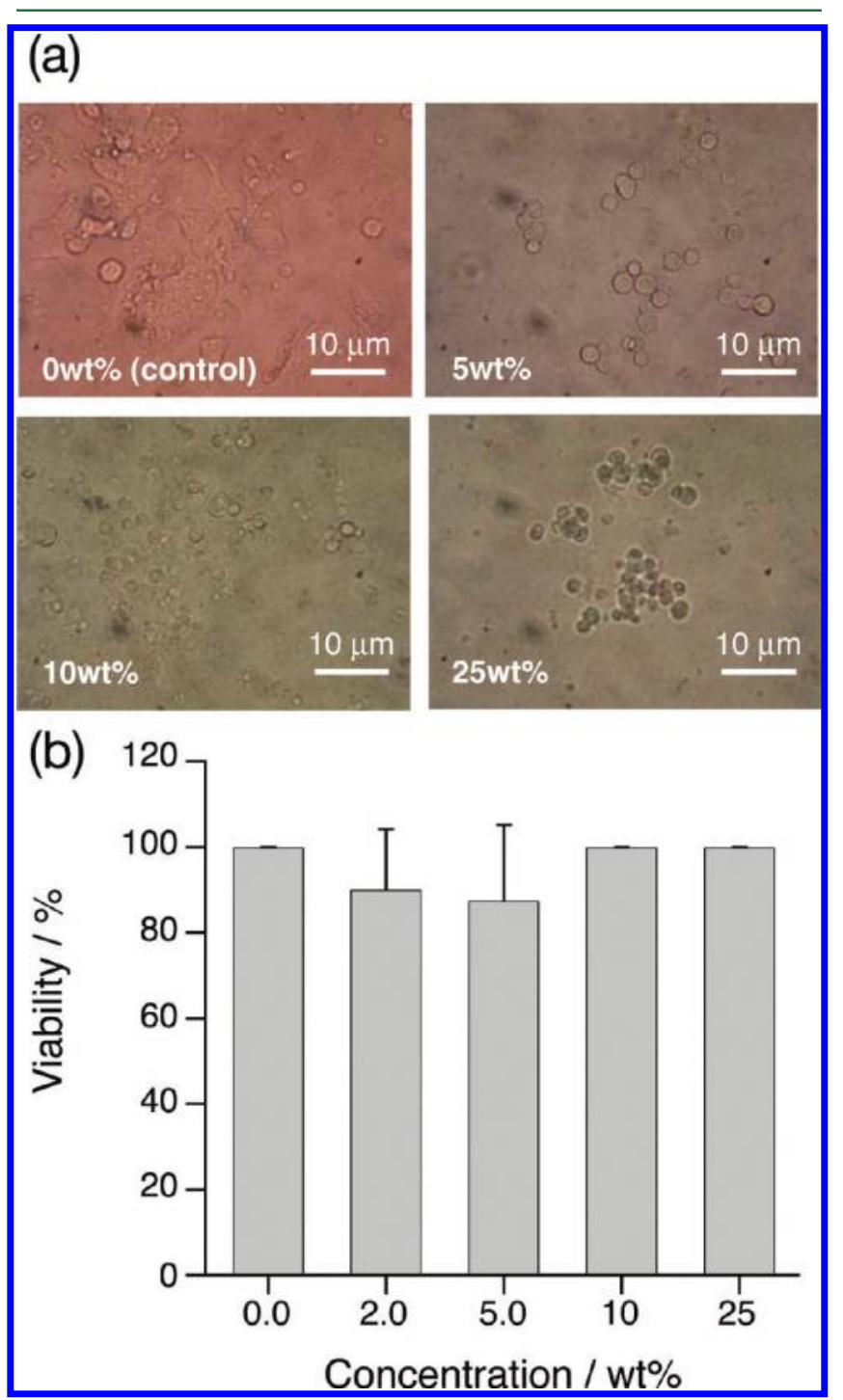

Figure 12. (a) Optical micrographs of trypan blue stained cells: control and incubated with 2,5 and $10 \mathrm{wt} \%$ of E10KDC18 polymer (indicated). There is no distinct change in morphology after a $48 \mathrm{~h}$ incubation with E10KDC18 polymer relative to the control. (b) The cytotoxicity profile of E10KDC18 polymer on murine catecholaminergic cell line derived from the B6/D2 F1 mouse after $48 \mathrm{~h}$ incubation, as measured with a trypan blue assay.

dose-dependent morphology change was observed after a 2-day incubation with E10KDC18 polymer relative to the control cells. The viability of the cells was measured after culturing for 2 days. As is evident from Figure 12b, the percentage viability of the cell is almost $100 \%$ within an experimental error. The cytotoxicity of the E10KDC18 polymer, therefore, is sufficiently low because there is no marked decrease in the cells interacting with E10KDC18 polymer for prolonged periods.

A degassed solution of $25 \mathrm{wt} \%$ E10KDC18 polymer in saline was injected into the vitreous cavity of 10 white rabbits through a 21-gauge cannula after core vitrectomy and fluid-gas exchange (Figure 13a). The injected E10KDC18 polymer solution exhibits rapid gelation in the vitreous cavity and does not leak out of the cavity. E10KDC18 gel exerts a hydraulic 


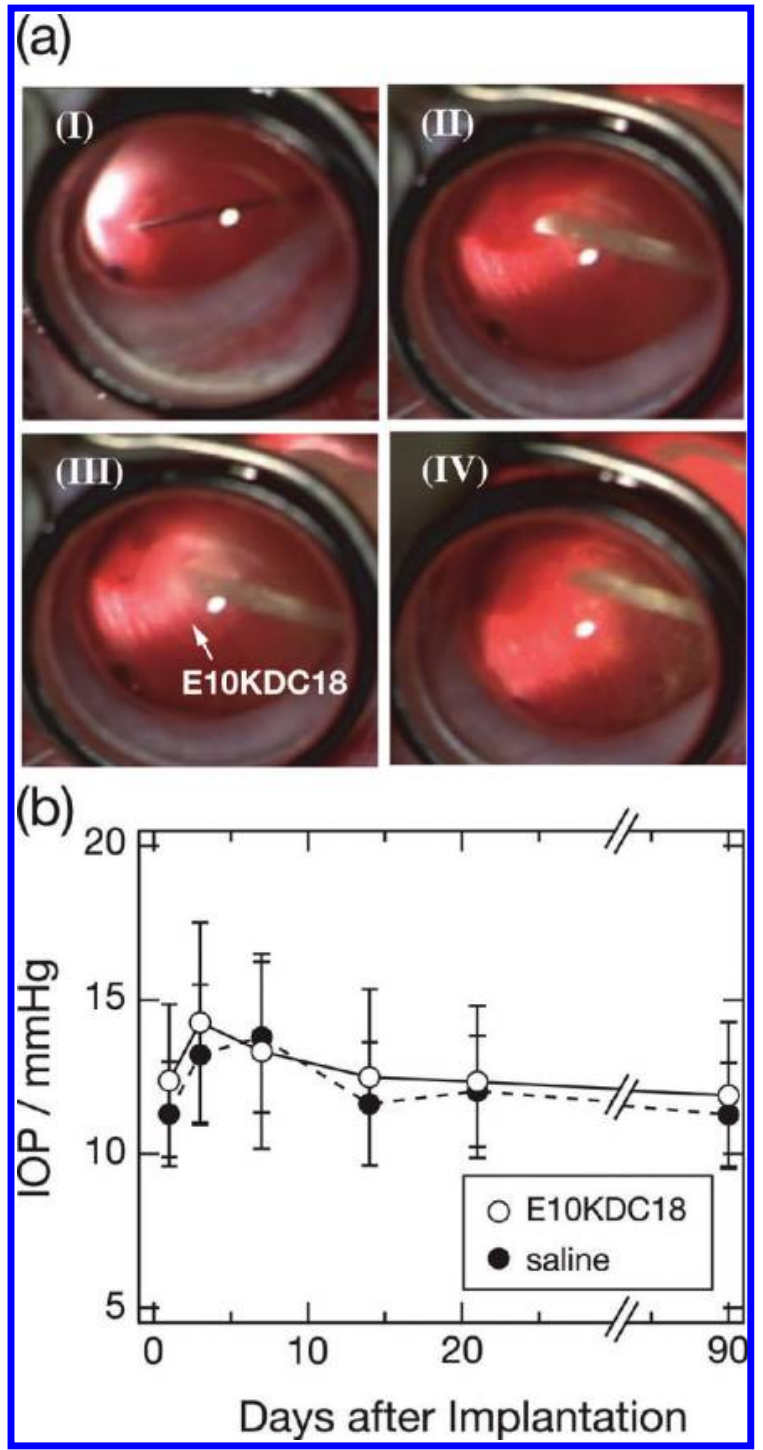

Figure 13. (a; i) Retinal breaks were created by using a needle. (ii) From the sclerotomy port, a 21-gauge cannula connected to a syringe filled with E10KDC18 polymer was inserted near the detached retina. (iii) $25 \mathrm{wt} \%$ E10KDC18 polymer in saline was injected into the vitreous cavity. (iv) E10KDC18 polymer forms a transparent gel and provides the tamponade effect. (b) Changes in intraocular pressure $(n$ $=10)$ after the implantation of the E10KDC18 gel. No significant differences are found between the E10KDC18 gel-injected eyes and the saline-injected eyes during the observation period.

pressure so as to provide tamponade to the retina against the pigment epithelium layer, which can alter the characteristics of the vitreous body in order to relax the preretinal traction and to prevent the formation of new vitroretinal traction due to cellular invasion laying down new scar tissue. No marked adverse reactions were detected in clinical observation, and E10KDC18 gel was maintained in the vitreous cavity without losing any transparency. Furthermore, the postoperative intraocular pressure (IOP) was maintained at a normal level throughout the observation period, and no difference from the contralateral eyes was observed (Figure 13b). This indicates that the E10KDC18 gel did not block the trabecular meshwork or other aqueous humor discharge pathways to cause elevation of IOP.
To confirm that the E10KDC18 gel effectively close the retinal break, we conducted the in vitro feasibility studies of intraocular use of $\mathrm{E} 10 \mathrm{KDC} 18$ gel in pig eyes. A retinal detachment with a hole was created in pig eyecup after the vitreous gel was removed. One of the eyecups was filled with 25 wt \% E10KDC18 gel, and the other was used as a control by filling with saline (Figure 14a). ${ }^{52}$ Retinal detachment occurs

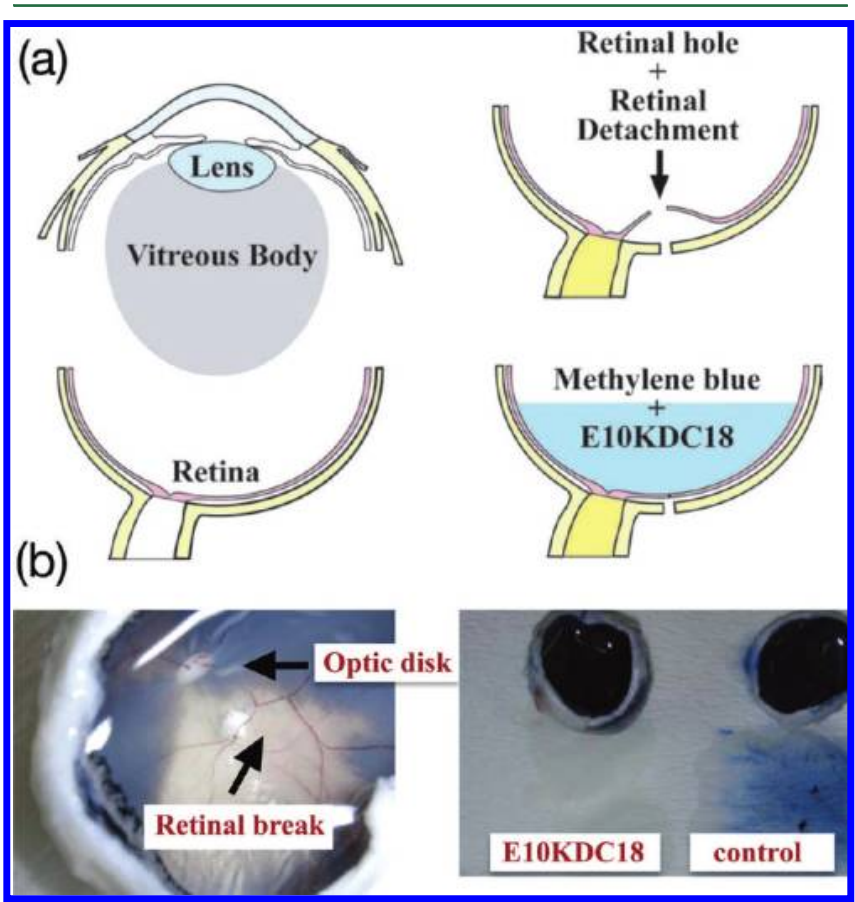

Figure 14. (a) Diagram of the process for observation of transport properties of E10KDC18 gel. (i) Anterior segments (cornea, iris, and lens) of a pig eye are excised. (ii) Retinal hole and retinal detachment are created. (iii) Eyecup was filled with 25 wt \% E10KDC18 gel. (b) Photograph for permeability of methylene blue in the E10KDC18 gel. (Left) The pig eyecup was filled with 25 wt \% E10KDC18 gel. Methylene blue does not seep into the underlying white paper. (Right) Control: The pig eyecup was filled with saline without closing fullthickness retinal hole. Underlying white paper was stained with methylene blue.

when subretinal fluid accumulates in the potential space between the neurosensory retina and the underlying retinal pigment epithelium (RPE). Whether or not significant fluid vitreous passes through a retinal break depends on the balance of forces acting at the edge of the break. Forces normally responsible for maintaining retinal attachment include negative pressure in the subretinal space created by the metabolic pump of the RPE and relatively higher oncotic pressure in the choroid. Retinal detachment occurs when forces favoring adherence of the retina are overwhelmed by forces promoting an accumulation of subretinal fluid. ${ }^{49}$ The liquid current from the vitreous should be controlled by well-developed network of the E10KDC18 gel. Dilute aqueous solution of methylene blue was applied to the eyecups to observe a transport property of E10KDC18 gel. Methylene blue did not seep onto the underlying white paper for the eyecups filled with E10KDC18 gel $2 \mathrm{~h}$ after application except a control (Figure 14b). When the water flows through a gel, gel network imposes a frictional resistance on the flowing water. The gel-solvent friction is primarily determined by the pore size of the network and the viscosity of water. Because the viscosity of water should 
be constant at fixed temperature, it is the spatial correlation length that determines the bulk frictional behavior of water in the E10KDC18 gel. In an inhomogeneous gel, some portions of the gel swell while the other portions shrink; therefore, the friction is expected to become smaller because the water passes through the swollen open space, avoiding the shrunken regions. Similar water flow process is also expected to observe in the gel that the network structure does not fully develop. Our result, therefore, indicate that the E10KDC18 gel forms the homogeneous gel network, which is allowed to swell freely and occupies the volume of the vitreous cavity. The E10KDC18 gel effectively close the retinal break, and proved to be superior for intraocular use as tamponade substance.

Histopathologic examination revealed that all layers of the retina were intact and no loss of tissue was evident (Figure

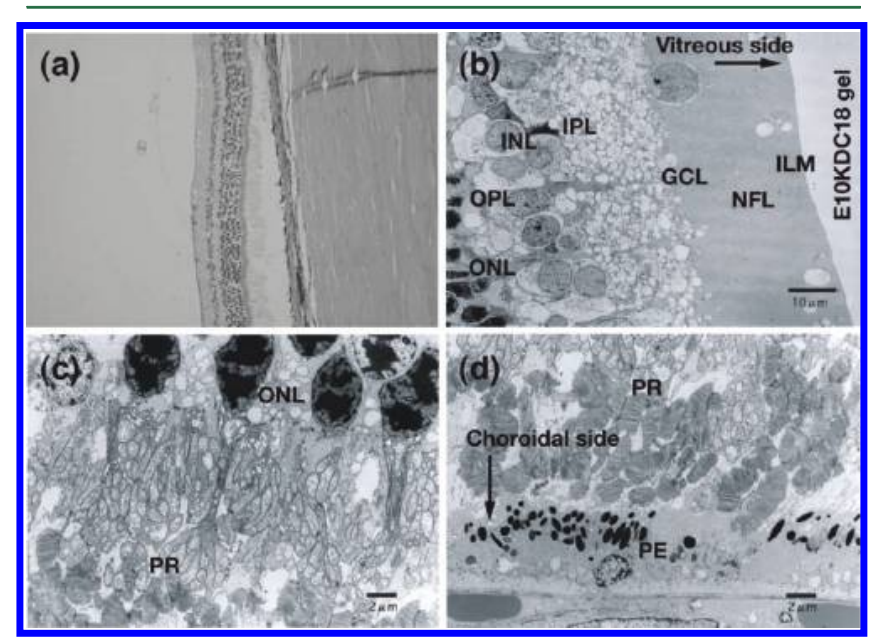

Figure 15. Electron micrographs of the retina in the E10KDC18 gelinjected eye. (A) All layers of the retina are intact. No remarkable change from (B) the internal limiting membrane to the outer plexiform layer, (C) the outer nuclear layer to the photoreceptor, and (D) the photoreceptor to the pigmented epithelium are found. ILM: internal limiting membrane; NFL: nerve fiber layer; GCL: ganglion cell layer; IPL: inner plexiform layer; INL: inner nuclear layer; OPL: outer plexiform layer; ONL: outer nuclear layer; OLM: outernal limiting membrane; PR: photoreceptor; PE: pigment epithelium.

15a). Disorganization of the inner layers of the retina, with loss of nerve fibers, loss of the parallel architecture of the glial columns, proliferation of glial cells, and infiltration of a large number of plasma cells and macrophages were not seen as shown in Figure $15 b-d$.

\section{SUMMARY AND CONCLUSION}

We report a novel biocompatible material for injectable vitreous substitute, composed of thermosensitive amphiphilic polymer, which exhibits rapid gelation to form a transparent gel in the vitreous cavity at body temperature. It is nontoxic, provides adequate support for the retina, and allows light to reach the sensory elements at the back of the eye. The postoperative intraocular pressure (IOP) was maintained at a normal level throughout the observation period, and no difference from the contralateral eyes was observed, which indicates that the E10KDC18 gel did not block the trabecular meshwork or other aqueous humor discharge pathways to cause elevation of IOP. All layers of the retina were intact and no loss of tissue was observed by histopathologic examination. The amphiphilic polymer exhibits mechanical stability by assembling to form highly interconnected hydrophobic domains, which leads to the constitution of a network structure. These results indicate that E10KDC18 gel demonstrates the feasibility of the development of an injectable vitreous substitute and has potential as a biomaterial suitable for long-term vitreous body replacement.

Recently, Nickerson et al. ${ }^{53}$ reported that the average steadystate moduli for bovine vitreous are $G^{\prime}=7 \pm 2 \mathrm{~Pa}$ (mean \pm SD) and $G^{\prime \prime}=2.2 \pm 0.6 \mathrm{~Pa}$, whereas for porcine vitreous are $G^{\prime}$ $=2.8 \pm 0.9 \mathrm{~Pa}($ mean $\pm \mathrm{SD})$ and $G^{\prime \prime}=0.7 \pm 0.4 \mathrm{~Pa}$. The mechanical properties of the human vitreous were more similar to the porcine vitreous than to the bovine vitreous. ${ }^{54}$ The moduli for the E10KDC18 hydrogel are approximately three orders magnitude higher than the porcine values. By varying the concentration of the E10KDC18 and/or the molecular weight of PEG, the rigidity of the final vitreous substitute could be tailored to match those of natural vitreous; however, weak gels are not expected to act as an effective vitreous tamponade agent to restore the volume and internal pressure of the ocular globe and to approximate the detached neurosensory retina to the retinal pigment epitherium for treatment of retinal tears, intravitreal hemorrhage, or retinal detachments. Weak gels are not also ideal due to the poor stability and the limited retention time in the vitreous cavity throughout the postoperative period, which preclude their long-term use. Although a cross-linked weak gel may improve retention time, a serious problem has to be considered: the shear stress produced by gel injection into the vitreous cavity cause significant deformation of the gel, possibly resulting in physical properties different from those displayed before injection. Specifically, the gel fragmentation due to shear stress during injection through small-gauge needles leads to detrimental consequences on the mechanical properties of the gel.

The E10KDC18 hydrogel fulfills the requirements for clinical use: clarity and transparency, biological and chemical inertness, refractive index similar to natural vitreous, sufficient rigidity to act as tamponade agent, nonabsorbable and nonbiodegradable characteristics, and ability to be injected through small-gauge needles. Although further analysis of long-term biocompatibility is needed, our preliminary results suggest that an application as vitreous substitute can be foreseen for E10KDC18 hydrogel.

\section{AUTHOR INFORMATION}

\section{Corresponding Author}

*E-mail: annaka@chem.kyushu-univ.jp.

\section{ACKNOWLEDGMENTS}

The work was partly supported by a Grant-in-Aid (No. 22350053) and a Grant-in-Aid for the Global COE Program, "Science for Future Molecular Systems" from the Ministry of Education, Culture, Science, Sports and Technology of Japan, and from the UNIK Synthetic Biology and DANSCATT programs of the Danish Research Council.

\section{REFERENCES}

(1) Vaugham, D.; Asbury, T. General Ophthalmology, 10th ed.; Lange Medical Publications: Maruzen Asia, 1983; 129-136.

(2) Andersen, H. L.; Sander, B. In Adler's Physiology of the Eye; Kaufman, P. L., Alm, A., Eds.; Mosby Co.: St. Louis, MO, 2003; pp293-315.

(3) Schepens, C. L., Neetens, A., Eds. The Vitreous and Vitreoretinal Interface; Springer-Verlag: Bergium, 1987.

(4) Swindle, K. E.; Ravi, N. Expert Rev. Ophthalmol. 2007, 2, 225.

(5) Baino, F. Acta Biomater. 2011, 7, 921. 
(6) Liesegang, T. J. Int. Ophthalmol. Clin. 1993, 33, 127.

(7) Fernandez-Vigo, J.; Refojo, M. F.; Verstraeten, T. Retina 1990, 10, 148.

(8) Lloyd, A. W.; Faragher, R. G. A.; Denyer, S. P. Biomaterials 2001, 22, 769.

(9) Mukai, N.; Lee, P. F.; Oguri, M.; Schepens, C. L. Can. J. Ophthalmol. 1975, 10, 391.

(10) Sugar, H. S.; Okamura, I. D. Arch. Ophthalmol. 1976, 94, 612.

(11) Eckardt, C.; Nicolai, U.; Czank, M.; Schmidt, D. Retina 1992, $12,17$.

(12) Kirchhof, B.; Tavakolian, U.; Paulmann, H.; Heimann, K. Graefe's Arch. Clin. Exp. Ophthalmol. 1986, 224, 34.

(13) Budde, M.; Cursifen, C.; Holbach, L. M.; Naumann, G. O. H. J. Ophthalmol. 2001, 131, 329.

(14) Couplaud, S. E.; Heimann, H.; Lee, W. R. In Retinology Today (In Memoriam Klaus Heimann); Kriegelstein, G. K., Ed.; Ad mamum medici: Gemmering, 2000; pp 37-42.

(15) Eller, A. W.; Friberg, T. R.; Mah, F. Am. J. Ophthalmol. 2000, $129,685$.

(16) Capone, J. A.; Aaberg, T. M. Curr. Opin. Ophthalmol. 1995, 6, 33.

(17) Dalton, P. D.; Chirila, T. V.; Hong, Y.; Jefferson, A. Polym. Gels Networks 1995, 3, 429.

(18) Hong, Y.; Chirila, T. V.; Vijayasekaran, S.; Shen, W.; Lou, X.; Dalton, P. D. J. Biomed. Mater. Res. 1998, 39, 650.

(19) Hong, Y.; Chirila, T. V.; Vijayasekaran, S.; Dalton, P. D.; Tahija, S. G.; Cuypers, M. J. H.; Constable, I. J. J. Biomed. Mater. Res. 1993, 30, 441.

(20) Vijayasekaran, S.; Chirila, T. V.; Hong, Y.; Tahija, S. G.; Dalton, P. D.; Constable, I. J.; McAllister, I. L. J. Biomater. Sci., Polym. Ed. 1996, 7, 695 .

(21) Chirila, T. V.; Hong, Y. Polym. Int. 1998, 46, 183.

(22) Aliyer, H. A.; Hamilton, P. D.; Ravi, N. Biomacromolecules 2005, 6, 204.

(23) Leone, G.; Consumi, M.; Aggravi, M.; Donati, A.; Lamponi, S.; Magnani, A. J. Mater. Sci: Mater. Med. 2010, 21, 2491.

(24) Lamponi, S.; Leone, G.; Consumi, M.; Greco, G.; Magnani, A. Int. J. Artif. Org. 2010, 33, 142.

(25) Lamponi, S.; Leone, G.; Consumi, M.; Greco, G.; Magnani, A. J. Biomater. Sci., Polym. Ed. 2011, DOI: 10.1163/092050611X554499.

(26) Davidorf, F. H.; Chambers, R. B.; Kwon, O. W.; Doyle, W.; Gresak, P.; Frank, S. G. Retina 1990, 10, 297.

(27) Pritchard, C. D.; Crafoord, S.; Andréasson, S.; Arnér, K. M.; O’Shea, T. M.; Langer.; Ghosh, F. K. Acta Biomater. 2011, 7, 936.

(28) Pham, Q. T.; Russel, W. B.; Thibeault, J. C.; Lau, W. Macromolecules 1999, 32, 2996.

(29) Pham, Q. T.; Russel, W. B.; Thibeault, J. C.; Lau, W. Macromolecules 1999, 32, 5139.

(30) Chilira, T. V. Prog. Polym. Sci. 1988, 23, 475.

(31) Peppas, N. A.; Langer, R. Science 1994, 263, 1715.

(32) Hamley, I. W. The Physics of Block Copolymers; Oxford Univ. Press: Oxford, 1988.

(33) Pedersen, J. S.; Geistenberg, M. Macromolecules 1996, 29, 1363.

(34) Kinnings, D. J.; Thomas, E. L. Macromolecules 1984, 17, 1712.

(35) Mortensen, K.; Schwahn, D.; Janssen, S. Phys. Rev. Lett. 1993, $71,1728$.

(36) Pusey, P. N.; van Megen, W. Nature 1986, 320, 340.

(37) Sebag, J. Prog. Polym. Sci. 1998, 23, 415.

(38) Worst, J. G. F.; Los, L. I. Cisternal Anatomy of the Vitreous; Kugler Publication: Amsterdam, 1995.

(39) Ponsioen, T. L.; Hooymans, J. M. M.; Los, L. I. Prog. Retinal Eye Res. 2010, 29, 580.

(40) Bishop, P. N. Prog. Retinal Eye Res. 2000, 19, 323.

(41) Jongebloed, W. L.; Worst, J. F. Doc. Ophthalmol. 1987, 63, 183.

(42) Kleppinger, R; Mischenko, N.; Theunissen, E.; Reynaers, H.; Koch, M. H. J.; Almdal, K.; Mortensen, K. Macromolecules 1997, 30, 7012.

(43) Keller, A.; Pedemonte, E.; Willmounth., F. M. Nature 1970, 225,538
(44) Ackerson, B. J.; Clark, N. A. Phys. Rev. A 1984, 30, 906.

(45) Koppi, K. A.; Tirrel, M.; Bates, F. S.; Almdal, K.; Mortensen, K. J. Rheol. 1994, 38, 999.

(46) Mortensen, K.; Brown, W.; Norden, B. Phys. Rev. Lett. 1992, 68, 2340.

(47) Mortensen, K.; Theunissen, E.; Kleppinger, R.; Almdal, K.; Reynaers, H. Macromolecules 2002, 35, 7773.

(48) Perreur, C.; Habas, J. -P.; François, J.; Peyrelaasse, J.; Lapp., A. Phys. Rev. E 2002, 65, 041802.

(49) Sebag, J. The Vitreous and Vitreoretinal Interface; Schepens, C. L., Neetens, A., Eds.; Springer-Verlag: Belgium, 1987; pp 37-58.

(50) Takeuchi, H.; Yoshikawa, M.; Kanda, S.; Nonaka, M.; Nishimura, F.; Yamada, T.; Ishizaka, S.; Sakaki, T. J. Neurosurg. 2001, 94, 775 .

(51) Jae-young, K.; Dennis, W. C. J. Neurosci. 1988, 8, 2153.

(52) Sueda, J.; Sakuma, T.; Nakamura, H.; Usumoto, N.; Okuno, T.; Arai, M.; Hirise, T. Inv. Ophthal. Vis. Sci. 2006, 47, 1142.

(53) Nickerson, C. S.; Park, J.; Kornfield, J. A.; Karageozian, H. L. J. Biomech. 2008, 41, 1840.

(54) Lee, B.; Litt, M.; Buchsbaum, G. Biorheology 1994, 32, 327. 